\title{
Le programme Leader+, du développement rural à l'innovation territoriale: exemples de deux initiatives locales en région Centre
}

\author{
Fabien Nadou \\ Fonds social européen en région Centre
}

\section{Introduction}

L'initiative communautaire Leader+, Liaison entre actions de développement de l'économie rurale, a pour vocation de soutenir des projets de développement rural exemplaires initiés par des acteurs locaux dans tous les secteurs d'activité du milieu rural afin de revitaliser des zones rurales et de créer des emplois.

Leader ${ }^{1} \mathrm{~s}^{\prime}$ inscrit dans la politique des fonds structurels européens ${ }^{2}$, et plus particulièrement dans les Programmes d'initiative communautaire (PIC) de l'Union européenne permettant d'expérimenter des approches nouvelles.

Leader I a marqué, en ce qui concerne le développement rural, le début d'une nouvelle approche intégrée reposant sur une base territoriale et faisant appel à la participation. Dans le cadre de Leader II, il a été fait plus largement usage de l'approche retenue pour Leader I, l'accent étant mis sur les aspects innovants des projets. Leader+ continue de jouer le rôle qui est le sien, à savoir celui d'un laboratoire destiné à encourager l'émergence et l'expérimentation, dans le contexte du développement intégré et du développement durable, d'approches nouvelles qui influenceront, compléteront ou renforceront la politique de développement rural dans l'Union européenne.

Notre exposé s'attardera sur deux exemples de projet du programme Leader+. Ce dernier vise à encourager la mise en œuvre de stratégies originales de développement durable intégrées dans les territoires ruraux.
Selon les termes de la communication aux États membres du 14 avril 2000, «Leader+ gardera sa fonction de laboratoire dans l'objectif de faire émerger et expérimenter de nouvelles approches de développement intégrées et durables qui doivent influencer, compléter et/ou renforcer la politique de développement rural dans la Communauté. »

Leader $+\mathrm{s}$ 'articule autour de trois volets ${ }^{3}$. Au total, en Europe, 5,046 milliards d'euros seront dépensés pour la période 2000-2006, dont 2,10 milliards d'euros à la charge de la section orientation du Fonds européen d'orientation et de garantie agricole (FEOGA), le reste provenant de contributions publiques et privées.

\section{Un programme de concertation et d'innovation territoriale}

L'objectif est de soutenir des initiatives et des projets de territoires ruraux visant à mettre en œuvre des stratégies de développement durable intégrées, le tout ayant pour objet d'expérimenter de nouvelles formes de valorisation du patrimoine culturel et naturel, de renforcer l'environnement économique afin de contribuer à la création d'emplois et à l'amélioration des capacités organisationnelles des acteurs locaux. L'élément fondamental constituant les programmes Leader+ est la coopération entre territoires, et l'originalité réside dans la mise en œuvre de démarches innovantes. Cinq éléments clés caractérisent Leader+ :

- l'approche territoriale (zonage adapté aux projets): cette action/projet entraîne la définition d'une politique de développement s'appuyant sur la si- 
tuation propre à un territoire, en termes de forces et de faiblesses;

- l'approche ascendante ou bottom-up (développement de projets endogènes) : cette action/projet vise à encourager la participation au processus décisionnel au niveau local pour tous les aspects du développement de la politique;

- le rôle de laboratoire (projets innovants) : même si le concept Leader et son application sur le terrain sont innovants en soi, l'initiative Leader+ insiste sur le fait que les actions elles aussi doivent être innovantes;

- la mise en réseau des partenaires (la concertation pour des projets partagés) : le Groupe d'action locale (GAL), composé d'acteurs publics et privés unis dans un partenariat, identifie une stratégie conjointe ainsi qu'un plan d'action local pour développer un territoire Leader;

- l'approche intégrée : les actions et les projets contenus dans le plan d'action local sont liés et coordonnés comme formant un tout cohérent.

\section{La mise en réseau vise à favoriser le dialogue et la concertation entre les partenaires du programme, les échanges d'expériences et de bonnes pratiques ainsi qu'à renforcer les partenariats.}

\section{Un territoire pertinent pour des projets cohérents}

La zone d'application est celle du territoire national, hors aires urbaines de plus de 50000 habitants (définition INSEE de 1990 avec population de 1999) et à l'exception de l'Île-de-France (Paris et sa région) Les projets sont mis en œuvre dans des territoires de dimension réduite à caractère rural qui ne couvrent pas la totalité d'un département, formant un ensemble homogène d'un point de vue physique (géographique), social et économique. Ils doivent représenter une cohérence et une masse critique suffisante en termes de ressources humaines et financières. L'unité du projet est appelée un Groupe d'action locale (GAL). En France, $140 \mathrm{GAL}$ ont été repérés pour développer des projets dans le cadre de Leader+ (carte 1).
La population du territoire concerné comprend entre 10000 et 100000 habitants pour les zones densément peuplées $\left(120 \mathrm{hab} / \mathrm{km}^{2}\right)$. Les bénéficiaires du programme sont un ensemble de partenaires composé au minimum de $50 \%$ de partenaires privés (partenaires économiques et sociaux, associations), porteurs d'un projet de territoire articulé autour d'une stratégie territoriale et responsables de sa mise en œuvre.

La mise en réseau vise à favoriser le dialogue et la concertation entre les partenaires du programme, les échanges d'expériences et de bonnes pratiques ainsi qu'à renforcer les partenariats. Un plan de développement atteste de la stratégie de développement territorial mise en place par les porteurs bénéficiaires, basé sur un partenariat représentatif et s'articulant autour de six thèmes fédérateurs, caractéristique de l'identité du territoire. Voici la répartition des GAL suivant les thèmes :

1. Utilisation de nouveaux savoir-faire et de nouvelles technologies de l'information : 15

2. Amélioration de la qualité de vie dans les zones rurales : 17

3. Valorisation des produits locaux : 19

4. Valorisation des ressources naturelles et culturelles : 58

5. Accueil de nouveaux acteurs locaux et d'entreprises : 18

6. Publics cibles, prioritaires (les jeunes ou les femmes) : 13

Nous prendrons deux exemples de projets Leader+ en région Centre. L'un porte sur l'initiative du Parc naturel régional (PNR) de la Brenne de favoriser la création d'entreprises par un public jeune en milieu rural; l'autre traite du développement d'un service de conseil en architecture rurale, paysage et environnement du Pays Loire Nature

Pour chacun des deux exemples, nous présenterons d'emblée les caractéristiques du territoire puis les aspects de l'opération et enfin les spécificités liées à la mise en réseau, aux partenariats développés et à la mise en perspective face aux fondamentaux d'une action Leader+. 


\section{Carte 1 - Répartition géographique des 140 GAL en France}

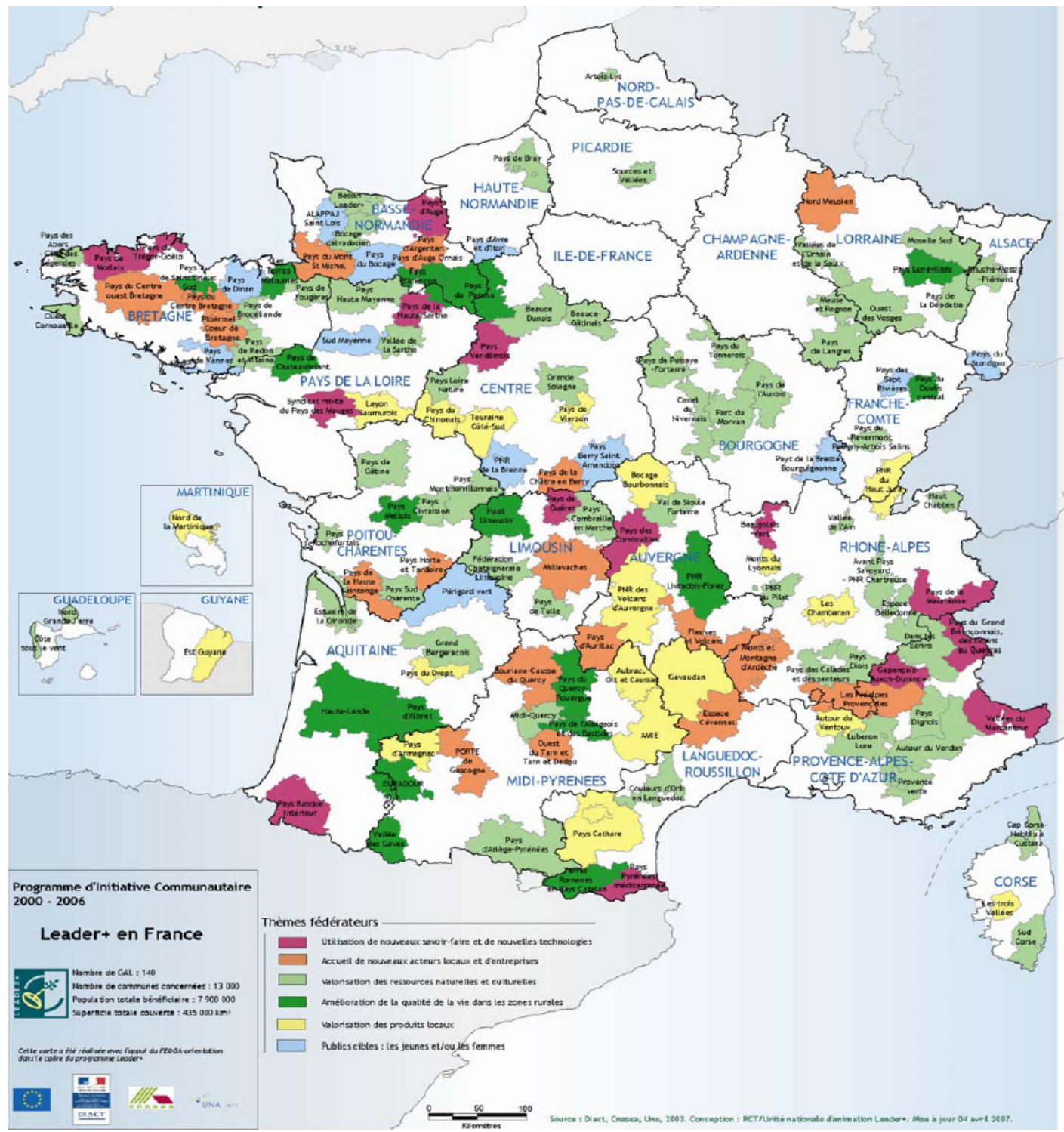

Source : Délégation interministérielle à l'aménagement et la compétitivité des territoires (DIACT), Unité nationale d'animation. Mise à jour avril 2007

\section{Favoriser la revitalisation économique d'un territoire}

Concours à la création et à la reprise d'entreprise dans le PNR de la Brenne

\section{Un territoire vieillissant}

La Brenne est une des principales zones humides continentales françaises, reconnue internationalement, se situant au carrefour de quatre régions historiques: Berry, Touraine, Poitou et Limousin, au sud-ouest de la région Centre. En 1999, lors du dernier recense- ment, le PNR de la Brenne comptait 31075 habitants ${ }^{4}$ dont $33,2 \%$ avaient plus de 60 ans, pour seulement $20 \%$ qui avaient moins de 20 ans. Le vieillissement de la population est significatif dans la Brenne qui n'échappe pas aux caractéristiques dans ce domaine des territoires ruraux en difficultés économiques.

Le manque d'activités économiques présentes sur le territoire, lié au manque d'emplois et aux débouchés professionnels qui en découlent, ne favorise pas la retenue des jeunes populations en demande de services, publics ou privés « offerts ». Dès lors que l'on s'éloigne du Blanc, sous-préfecture de l'Indre, ce constat se 
vérifie et les conséquences sur le dynamisme local deviennent évidentes. En outre, le tissu économique et d'entreprises de la Brenne est presque exclusivement composé de PME artisanales et commerciales, avec une moyenne d'âge des dirigeants et chefs d'entreprises qui suit l'évolution vieillissante de la population locale. En conséquence, le taux de reprise des entreprises locales est faible et de nombreux commerces ont du mal à trouver des repreneurs.

À partir de ces éléments diagnostiqués et constatés par les acteurs du territoire, la stratégie de ces derniers s'est tournée vers des propositions d'actions visant à soutenir les initiatives des jeunes de moins de 36 ans, public cible contribuant au dynamisme du territoire, notamment au travers du programme « Brenne Initiatives Jeunes ». Les constats socio-économiques précités ont motivé le lancement de l'opération « concours à la création et à la reprise d'entreprises », action spécifique financée et mise en place dans le cadre de Leader+. Deux objectifs majeurs sont poursuivis dans cette action :

- stimuler la création ou la reprise d'entreprise par les jeunes issus du territoire ou souhaitant s'y établir;

- faire découvrir ce qu'est la création d'entreprise auprès des jeunes du territoire, notamment via le concours junior réalisé en partenariat avec le Lycée du Blanc.

\section{La volonté de rapprocher les activités du Parc naturel régional et de la création d'entreprise}

Les responsables du Parc de la Brenne ont voulu mettre l'accent sur la compatibilité entre les activités de développement économique local et les activités « classiques » environnementales d'un parc naturel régional. Ils ont insisté sur la volonté de rapprocher un public jeune avec le contexte naturel et rural qui caractérise le PNR. Cette volonté s'est matérialisée via l'organisation du concours à la création et reprise d'entreprises dans le cadre d'une candidature à un financement Leader+, dont la mise en œuvre s'appuie sur les éléments fondamentaux décrits en introduction.

Le PNR représente une zone géographique pertinente pour le développement de projets locaux de territoire, reconnu et labellisé comme un Groupe d'action locale parmi les 140 en France. Depuis 1992, le PNR de la Brenne a confié les missions économiques ${ }^{5}$ à 1 'Association de développement économique du parc (ADEP). Elle réunit différents acteurs et partenaires impliqués dans le développement économique du territoire, dont la gestion revient à un conseil d'administration et un bureau représentant les différents membres au sein de trois collèges (collectivités territoriales, experts et chefs d'entreprises du parc). Cette structure est devenue le véritable interlocuteur privilégié des initiatives locales et permet d'être l'interface opérationnelle du Parc pour les porteurs de projets locaux et ainsi de poursuivre les objectifs de dynamiser le territoire.

L'outil ADEP permet, de ce fait, le développement et l'opérationnalité de projets endogènes au territoire du PNR de la Brenne, symbolisé par le programme d'ensemble « Brenne initiative ». Le concours à la création et à la reprise d'entreprise est porté par l'ADEP, mâ̂tre d'ouvrage de l'opération lancée en fin d'année 2003 pour se clôturer en avril 2004. Ce concours s'adressait, d'une part, à tous les jeunes de moins de 36 ans présentant des projets dans le périmètre du parc et, d'autre part, à des lycéens pour constituer des dossiers fictifs et de simulation. Le dispositif bénéficie de prestations techniques de la part du PNR et des chambres consulaires partenaires du projet (des conventions de partenariat fixant le nombre d'heures passées par les techniciens).

Les bénéficiaires doivent avoir un projet de création ou de reprise sur le territoire du parc, et être ressortissant de l'Union européenne. Des critères préétablis ont guidé les porteurs : la contribution et sa propension au dynamisme local, la cohérence entre le profil du porteur et le projet développé, l'originalité du projet, la prise en compte de l'environnement (matériaux utilisés, gestion des déchets, etc.). Sur le volet «concours pour les lycéens » (projet fictif tels que cybercafé, écuries automobile, location immobilière), ces derniers ont suivi chaque mercredi des formations véritablement inscrites dans une démarche de création d'activité : définition du projet, étude de faisabilité, étude des compétences nécessaires à mobiliser et à développer, montage juridique, montage financier. Pour les deux volets du concours, des prix récompensent les projets retenus : deux fois 10000 euros pour les projets «réels » et 1500 euros pour un des groupes de lycéens. Les différentes étapes du projet ont été les suivantes : 
- 2001 : idée développée dès la conception du dossier de candidature au programme Leader+;

- 2001-2003 : conception du projet «concours» (modalités, partenaires techniques à mobiliser);

- 2003 : recherche active des partenaires financiers, lancement de l'opération;

- 2004 : remise des prix des premiers lauréats.
Compte tenu des délais imposés par les procédures d'appel d'offres dans le cadre des fonds structurels européens, du temps nécessaire à la mobilisation des partenaires et de quelques difficultés pour compléter le plan de financement, un projet comme celui-ci, d'une durée relativement courte (six mois), s'étend sur trois années complètes entre la conception et la réalisation finale. C'est dire si le développement local est bien une affaire de temps et de patience.

\section{Tableau 1 - Plan de financement du projet}

\begin{tabular}{|c|c|c|c|}
\hline Recettes & Montants en $€$ & Dépenses & Montants en $€$ \\
\hline FEOGA & 46090,58 & Communication & 26800 \\
\hline Contributions privées & 16557,58 & Intervention chambre consulaire & 38800 \\
\hline Chambres consulaires & 21340,00 & Frais de personnel & 15008,69 \\
\hline \multirow{3}{*}{ Autofinancement PNR Brenne } & 8193,00 & Intervention technicien du PNR & 8193 \\
\hline & & PNR & 2129,47 \\
\hline & & Prix du concours junior & 750 \\
\hline TOTAL & 92181,16 & TOTAL & 92181,16 \\
\hline
\end{tabular}

Source : PNR de la Brenne

Les partenaires financiers sont: Commission européenne (Leader+), Caisse des dépôts et consignations, Chambre de métiers de l'Indre, Chambre de commerce de l'Indre, Centre E. Leclerc (grande distribution) du Blanc.

L'objectif était de recueillir une vingtaine de dossiers de candidatures à la création et à la reprise. L'initiative a permis de mobiliser une trentaine de dossiers sur le volet « réels » et une vingtaine de lycéens sur le volet « fictifs ». Au total, les résultats succincts sont :

- 31 dossiers de candidatures déposés;

- 21 entreprises ont réellement été créées ou reprises;

- 38 emplois ont été créés et/ou maintenus.

À ce jour, les 21 entreprises créées ou reprises grâce à ce programme sont toujours en activité.

\section{Des pratiques communes formalisées}

Le partenariat s'est structuré autour de pratiques communes identifiées et formalisées. L'opération a permis de renforcer le partenariat déjà présent sur le territoire dans le domaine de l'accueil et du conseil aux porteurs de projet, notamment via les actions de l'ADEP.

La politique de développement économique du PNR de la Brenne repose principalement sur ses relations étroites et régulières avec l'ADEP et les chambres consulaires, notamment dans la préparation d'événements tels que le salon "Projet en campagne» ou bien dans l'implication de techniciens experts sur les projets de développement dans le cadre de l'attribution du fonds d'avance remboursable mis en place localement.

L'appartenance du PNR de la Brenne à la Fédération nationale des PNR a permis de capitaliser et d'échanger sur l'expérience déjà mise en place par le PNR du Livradois-Forez (au cœur du Massif Central, aux portes de Clermont-Ferrand et qui englobe Thiers capitale de la coutellerie, identifié comme $\mathrm{SPL}^{6}$ par la DATAR, et Ambert, célèbre pour sa fourme) qui avait auparavant organisé ce type de concours, mais pas spécifiquement destiné à un public jeune.

La valeur ajoutée du partenariat repose sur une identification plus précise des actions du PNR de la Brenne et de l'ADEP en faveur du développement 
économique du territoire. La réflexion commune et l'approche concertée dans la mise en place de ce projet permettent d'agir au plus près des préoccupations du territoire et de ses spécificités socio-économiques.

Compte tenu des résultats obtenus, les acteurs du territoire ont poursuivi l'action avec l'organisation d'une deuxième édition du concours s'adressant à un nombre plus important d'entreprises privées impliquées dans le financement des prix lauréats. Les partenariats financiers privés ont changé entre les deux éditions, mais le travail effectué avec les chambres consulaires a été maintenu, notamment dans le cadre de l'avance remboursable. De même, un projet d'un fonds d'avance remboursable spécifiquement dédié aux jeunes créateurs d'entreprises a été mise en place. Ces partenariats apparaissent transférables sur tout territoire organisé ayant une action dans le domaine du développement économique.

La réflexion commune et l'approche concertée dans la mise en place de ce projet permettent d'agir au plus près des préoccupations du territoire et de ses spécificités socio-économiques.

\section{Innovation, réactivité et projets locaux face à des enjeux globaux}

\section{Création d'un service de conseil en architecture rurale, paysage et environnement sur le Pays Loire Nature (SCARPE ${ }^{7}$ )}

\section{Caractéristiques et contexte du territoire d'appartenance}

Le territoire est composé de 36600 habitants $^{8}$ et se situe dans le département d'Indre-et-Loire à l'ouest de la région Centre, à 35 kilomètres de Tours, menant sur la route de Nantes. L'industrie et la construction (51\% des emplois) ainsi que l'agriculture (17\%) représentent les principaux secteurs d'activité. Le secteur des services demeure sous-représenté. L'agriculture spécialisée est assez présente avec la culture des arbres fruitiers du verger. Les objectifs de l'opération sont une réponse à des enjeux éco-environnementaux globaux passant par :

- la sensibilisation de la population locale, des collectivités et des acteurs économiques aux spécifici- tés environnementales du territoire pour développer une gestion environnementale collective autour du paysage. La qualité des paysages et l'amélioration du cadre de vie sont des éléments d'appropriation et de valorisation du territoire par les acteurs locaux;

- une gestion cohérente, durable et réfléchie du patrimoine bâti et paysager, de l'énergie, de l'urbanisme et de l'environnement en général.

\section{L'environnement, un enjeu, au cœur du projet de territoire}

Dans un souci de cohérence territoriale, le Pays Loire Nature, en partenariat avec les communautés de communes ${ }^{9}$, a souhaité créer un Service rural de conseil en architecture, urbanisme, paysage et environnement (SCARPE). La maîtrise d'ouvrage du projet revient au syndicat mixte du Pays Loire Nature. Ce service est lancé en parallèle de la réflexion et des études engagées au titre de l'élaboration du Schéma de cohérence territorial (SCOT) qui doit coordonner les interventions locales en lien avec le développement architectural, paysager et environnemental local. Le service proposé permettra des actions dans de nombreux domaines $^{10}$ :

- En matière d'architecture - cadre de vie :

- de conseiller et de sensibiliser l'ensemble des acteurs et des habitants du territoire sur les formes architecturales ainsi que les matériaux les mieux adaptés pour les restaurations du patrimoine local ou les nouvelles constructions par le biais de permanences et de conseil d'un architecte et la publication de brochures et de guides;

- de conseiller et de sensibiliser l'ensemble des acteurs et des habitants à la prise en compte du bois dans les constructions;

- de promouvoir les énergies renouvelables, les outils de gestion de l'eau et des déchets dans le bâti.

- En matière de paysage :

- de conseiller et de sensibiliser sur les paysages au sens large par la publication de brochures et de guides remis lors des demandes de permis de construire; 
- de mettre en place des conseils personnalisés pour les collectivités et les particuliers (sur les essences naturelles locales, sur la gestion des espaces verts);

- de réaliser une charte paysagère à l'échelle du Pays Loire Nature et de mettre en place des outils pour son application.

- En matière d'énergie/environnement :

- d'organiser, pour les particuliers, des rencontres-conseils avec l'espace Info-Énergie et ainsi sensibiliser à une meilleure prise en compte des énergies renouvelables et des économies d'énergie dans le quotidien. En parallèle, le service rural développera ou adaptera des outils pour sensibiliser l'ensemble des acteurs du territoire sur ces problématiques;

- de renforcer le conseil sur les énergies et l'environnement au niveau des collectivités et des professionnels ainsi que de suivre les procédures environnementales (Natura 2000, Espaces Naturels Sensibles);

- de servir de relais entre les acteurs du territoire et les structures existantes travaillant sur des questions liées à l'énergie, l'environnement, le patrimoine, l'urbanisme, les transports (Chambre d'Agriculture, Maison Paysanne, Fondation du patrimoine, PNR...).

Contrairement à l'initiative développée dans la Brenne, ici aucun fonds privé n'alimente le fonctionnement de l'opération. Le partenariat financier est assuré par trois communautés de communes.

\section{Tableau 2 - Partenariat financier}

\begin{tabular}{lc} 
Plan de financement & Montant en $€$ \\
\hline FEOGA-0 & 43498 \\
Fonds publics & 34575 \\
Fonds privés & 0 \\
\hline TOTAL & $\mathbf{7 8 ~ 0 7 3}$
\end{tabular}

Source : Pays Loire Nature

Deux phases sont prévues dans le projet: année 1, lancement du SCARPE (du $1^{\text {er }}$ janvier au 31 décembre 2007); année 2, bilan et évaluation du service (du $1^{\text {er }}$ janvier au 30 septembre 2008). Afin d'organiser une bonne coordination entre les structures intervenantes, un bon suivi des missions de ce nouveau service et d'assurer la gestion des activités projetées, le service sera sous la responsabilité d'un chargé de mission qui coordonnera les missions de conseils, de sensibilisation et d'information.

\section{Un partenariat nécessairement basé sur des compétences techniques}

L'ensemble des missions du SCARPE peut être assuré grâce à la participation d'intervenants extérieurs, de bureaux d'études, de stagiaires dans le cadre de partenariats avec des écoles. La mise en place d'un partenariat avec une école spécialisée sur la thématique paysagère est organisée, pour les opérations de sensibilisation et de communication, sous différentes formes (plaquette grand public, plaquette lors des demandes de permis de construire, expositions...). L'école participera à la mise en place d'une charte paysagère sur l'ensemble du Pays Loire Nature. Ce service est le résultat d'un partenariat structuré par des pratiques communes. Ce projet, dans sa mise en œuvre, mobilise une partie du public cible (ici les communes, les communautés de communes) et le bénéficiaire final tout au long de l'action, avec les mêmes responsabilités qu'un autre acteur (concertation, réseau). Il y a là un véritable partage intégral de la décision et de la responsabilité au niveau local. Cette initiative permet l'émergence d'un nouveau service qui tient compte des spécificités locales et les intègre, permettant également de mêler entre elles les ressources humaines, naturelles et financières du territoire, le tout conduisant à une meilleure exploitation de ses capacités endogènes (approche ascendante). Ce projet contribue à la structuration du projet de territoire dans sa globalité et la mise en œuvre des mesures clés stratégiquement adoptées par les acteurs du Pays (approche intégrée).

La création d'un tel service de conseil en architecture rurale, paysage et environnement à l'échelle d'un Pays est une opération nouvelle pour le territoire et peu répandue au niveau régional ou national (innovation territoriale).

\section{Conclusion}

La plus-value du programme Leader+ est reconnue par les acteurs de territoire sur trois points essentiels : 
- un partenariat public-privé fonctionnant sur du concret et favorisant la mise en réseau des acteurs. Cela tient notamment au fait de réunir des personnes de divers horizons et de favoriser ainsi l'inter-connaissance des acteurs d'un territoire;

- la conjonction de la gestion locale d'une subvention globale et d'une animation renforcée. Sont mis en avant la réactivité financière, la souplesse dans le choix des projets à soutenir et la responsabilisation collective des acteurs locaux que soustend un tel système;

- l'effet levier du financement européen. Leader+ permet de financer des opérations qui n'auraient pu être soutenues sans l'intervention de crédits européens; celle-ci tend à rassurer les autres financeurs (État, conseil régional, conseil général...).

\section{Notes et références}

$1 \quad$ Leader I (1987-1993); Leader II (1994-1999); Leader+ (2000-2006); « Axe Leader » (2007-2013).

2 Quatre fonds structurels guident la politique d'intervention de l'Union européenne: Fonds social européen (FSE), Fonds de développement régional (FEDER), Fonds européen d'orientation et de garantie agricole (FEOGA-0) et Instrument financier d'orientation de la pêche (IFOP).

3 Voir la description des trois volets en annexe.

4 Source : INSEE, recensement de la population française 1999.

5 Le PNR de la Brenne dispose d'un site Internet avec un portail d'accès et une rubrique dédiés aux services économiques délivrés sur le territoire: www.parc-naturelbrenne.fr.

6 Système productif local, territoire d'activité économique inspiré des districts industriels « marshalliens », défini par la DATAR (DIACT depuis 2004).

7 Projet en cours de réalisation sur le Pays Loire Nature, débuté en 2007.

8 Source : INSEE, recensement de la population française, 1999.
9 En général et pour une explication simple, un Pays est composé de plusieurs communautés de communes, qui siègent à l'assemblée du syndicat de Pays

10 Plan d'action défini par le maître d'ouvrage. Source : Pays Loire Nature.

11 Communication de la Commission aux États membres du 14 avril 2000 fixant les orientations pour l'initiative communautaire concernant le développement rural (Leader+) 2000/C 139/05.

\section{Annexe \\ Description des trois volets d'intervention du programme Leader $+{ }^{11}$}

Le volet 1 est mis en œuvre par l'intermédiaire des groupes d'action locale (GAL) sélectionnés grâce à une procédure ouverte fondée sur les critères exposés dans les programmes. Parmi ces critères, il faut mentionner notamment la ruralité des territoires concernés, leur homogénéité physique, économique et sociale et le caractère intégré et innovant des plans de développement. Les partenaires économiques et sociaux et les associations doivent représenter au moins $50 \%$ du partenariat local, dont la pertinence et l'efficacité entrent également en ligne de compte.

Le volet 2 s'applique aux collectivités territoriales rurales sélectionnées dans le cadre du volet 1 (stratégies intégrées de développement rural). Un GAL coordinateur est responsable de sa bonne exécution. Le GAL est explicitement chargé d'encourager et de soutenir les collaborations entre les différents territoires ruraux et doit coordonner les projets de coopération existant entre des régions du même État membre (coopération inter-territoriale) ou entre des régions appartenant à au moins deux États membres (coopération transnationale). Des coopérations sont également possibles avec des pays non membres de l'Union européenne.

Il existe une Unité nationale d'animation dans chaque État membre chargée de recueillir, d'analyser et de distribuer à l'échelon national les informations relatives aux meilleures pratiques. Elle est aussi chargée d'organiser les échanges d'expériences et de savoir-faire et de fournir une assistance technique dans le cadre des coopérations (voir ci-dessus). Une participation active au réseau est obligatoire pour les groupes d'action locale Leader+.

\begin{tabular}{lcc} 
Plan de financement du programme Leader+ 2000-2006 en Europe & Montant en millions $€$ & Part en \% \\
\hline Volet 1: Aide en faveur de stratégies intégrées expérimentales de & 4377,6 & 86,75 \\
& & \\
développement territorial, faisant appel aux volets de la base & 504,8 & 10,00 \\
Volet 2: Soutien de la coopération entre collectivités territoriales rurales & 68,7 & 1,36 \\
Assistance technique & 95,4 & 1,89
\end{tabular}

